\title{
On control laws for discrete linear repetitive processes with dynamic boundary conditions
}

\author{
Lukasz Hladowski • Eric Rogers • \\ Krzysztof Galkowski • Virendra R. Sule
}

Received: 4 April 2007 / Revised: 4 November 2007 / Accepted: 6 November 2007 /

Published online: 20 December 2007

(C) Springer Science+Business Media, LLC 2007

\begin{abstract}
Repetitive processes are characterized by a series of sweeps, termed passes, through a set of dynamics defined over a finite duration known as the pass length. On each pass an output, termed the pass profile, is produced which acts as a forcing function on, and hence contributes to, the dynamics of the next pass profile. This can lead to oscillations in the sequence of pass profiles produced which increase in amplitude in the pass-to-pass direction and cannot be controlled by application of standard control laws. Here we give new results on the design of physically based control laws for so-called discrete linear repetitive processes which arise in applications areas such as iterative learning control.
\end{abstract}

Keywords Linear repetitive processes - Dynamic boundary conditions ·

Behavioral approach

\section{Introduction}

The unique characteristic of a repetitive, or multipass, process is a series of sweeps, termed passes, through a set of dynamics defined over a fixed finite duration known as the pass length. On each pass an output, termed the pass profile, is produced which acts as a forcing function on, and hence contributes to, the dynamics of the next pass profile. This, in turn, leads to the unique control problem in that the output sequence of pass profiles generated can contain oscillations that increase in amplitude in the pass-to-pass direction.

\footnotetext{
L. Hladowski $(\bowtie) \cdot$ K. Galkowski

Institute of Control and Computation Engineering, University of Zielona Gora, ul. Podgorna 50, 65-246 Zielona Gora, Poland

e-mail: L.Hladowski@issi.uz.zgora.pl

E. Rogers

University of Southampton, Southampton, SO17 1BJ, UK

V. R. Sule

Computational Laboratories Ltd., Pune, India
} 
To introduce a formal definition, let $\alpha<+\infty$ denote the pass length (assumed constant). Then in a repetitive process the pass profile $y_{k}(p), 0 \leq p \leq \alpha-1$, generated on pass $k$ acts as a forcing function on, and hence contributes to, the dynamics of the next pass profile $y_{k+1}(p), 0 \leq p \leq \alpha-1, k \geq 0$.

Physical examples of these processes include long-wall coal cutting and metal rolling operations (Edwards 1974). Also in recent years applications have arisen where adopting a repetitive process setting for analysis has distinct advantages over alternatives. Examples of these so-called algorithmic applications include classes of iterative learning control schemes (Moore et al. 2005) and iterative algorithms for solving nonlinear dynamic optimal control problems based on the maximum principle (Roberts 2000).

Attempts to control these processes using standard (or 1D) systems theory/algorithms fail (except in a few very restrictive special cases) precisely because such an approach ignores their key features, i.e. (i) information propagation occurs from pass-to-pass and along a given pass, and (ii) the initial, or boundary, conditions are reset before the start of each new pass. Of particular interest in this paper is the case when the initial conditions on each pass are an explicit function of points along the previous pass-so-called dynamic boundary conditions. In particular, it is known that such boundary conditions alone can cause instability (Owens and Rogers 1999) (the analysis in this paper is for processes which are the natural counterparts of those considered here). Here we will also show how dynamic boundary conditions can be used in stabilization in a manner akin to boundary control for partial differential equations.

In seeking a rigorous foundation on which to develop a control theory for these processes, it is natural to attempt to exploit structural links which exist between, in particular, the class of so-called discrete linear repetitive processes and 2D linear systems described by the extensively studied Roesser or Fornasini Marchesini state-space models (see the original references cited in, for example Rogers and Owens (1992)). Dynamic boundary conditions have no equivalent in these 2D discrete linear systems state-space models and hence systems theory developed for them cannot be applied.

The next section gives the necessary background to the analysis in this paper.

\section{Preliminaries}

The state-space model of the discrete linear repetitive processes considered in this paper has the following form over $0 \leq p \leq \alpha-1, k \geq 0$,

$$
\begin{aligned}
x_{k+1}(p+1) & =A x_{k+1}(p)+B u_{k+1}(p)+B y_{k}(p) \\
y_{k+1}(p) & =C x_{k+1}(p)+D_{0} y_{k}(p)
\end{aligned}
$$

where on pass $k x_{k}(p)$ is the $n \times 1$ state vector, $y_{k}(p)$ is the $m \times 1$ pass profile vector, and $u_{k}(p)$ is the $r \times 1$ vector of control inputs.

To complete the process description, it is necessary to specify the boundary conditions, i.e. the initial pass profile and the pass state initial vector sequence. The simplest form of these is

$$
\begin{aligned}
x_{k+1}(0) & =d_{k+1}, \quad k \geq 0 \\
y_{0}(p) & =y(p), 0 \leq p \leq \alpha-1
\end{aligned}
$$

where $d_{k+1}$ is an $n \times 1$ vector with constant entries and $y(p)$ is an $m \times 1$ vector whose entries are known functions of $p$. In some cases, however, this form of $x_{k+1}(0), k \geq 0$, must be extended to adequately model the underlying process dynamics (even for initial simulation 
and/or control analysis). Instead, it is necessary to consider a state initial vector sequence which is an explicit function of the previous pass profile. The most general case is

$$
x_{k+1}(0)=d_{k+1}+\sum_{j=0}^{\alpha-1} J_{j} y_{k}(j)
$$

where $J_{j}$ is an $n \times \mathrm{m}$ matrix. A state initial vector sequence of this form is required in the optimal control application (Roberts 2000).

The stability theory (Rogers and Owens 1992) for linear repetitive processes is based on an abstract model in a Banach space setting which includes a wide range of examples as special cases, including those considered here. In terms of their dynamics it is the pass-topass coupling (noting again the unique control problem for them) which is critical. This is of the form $y_{k+1}=L_{\alpha} y_{k}$, where $y_{k} \in E_{\alpha}\left(E_{\alpha}\right.$ a Banach space with norm $\left.\|\cdot\|\right)$ and $L_{\alpha}$ is a bounded linear operator mapping $E_{\alpha}$ into itself.

Two concepts of stability can be defined but here it is the first of these, so-called asymptotic stability which is considered. This holds if, and only if there exist numbers $M_{\alpha}>0$ and $\lambda_{\alpha} \in(0,1)$ such that $\left\|L_{\alpha}^{k}\right\| \leq M_{\alpha} \lambda_{\alpha}^{k}, k \geq 0$ (where $\|\cdot\|$ also denotes the induced operator norm) and can be interpreted as bounded-input bounded-output stability over the finite and constant pass length. The second stability property, so-called stability along the pass, is stronger in the sense that it demands this boundedness property uniformly, i.e. independent of the pass length.

Asymptotic stability is a necessary condition for stability along the pass and there are cases where only it can be achieved or is required. For example, in the optimal control application (Roberts 2000) only asymptotic stability can ever hold (see also the discussion in the next but one paragraph).

It is of interest to relate this theory to a physical example in the form of long-wall coal cutting (see, e.g. the original references given in Rogers and Owens 1992) where the pass profile is the thickness (relative to a fixed datum) of the coal left after the cutting machine has moved along the pass length, i.e. the coal face. The stability problem here is caused by the effects of the machine's weight as it rests on the previous pass profile during the cutting of the next pass profile. The resulting undulations can be very severe and result in productive work having to stop to enable them to be removed. Asymptotic stability here means that after a sufficient number of passes have elapsed the profile produced on each successive pass is the same, i.e. convergence in the pass to pass (i.e. $k$ ) direction and this converged value is termed the limit profile. However, this limit profile can contain growth along it, i.e. nonconvergence in the $p$ direction. Stability along this pass prevents this from happening by demanding convergence in both directions.

It is important to note again that asymptotic stability is often all that is required or indeed can be achieved. For example, in the optimal control example (Roberts 2000), it is never possible for the resulting iterative solution algorithm to be stable along the pass. In such cases there is a requirement to understand the mechanism(s) by which an example can be asymptotically unstable and also to determine if it is possible to enforce this property by the introduction of suitable control action. This is the subject area which is addressed in the rest of this paper.

Asymptotic stability in the presence of boundary conditions of the form (2) holds (Rogers and Owens 1992) if, and only if, $r\left(D_{0}\right)<1$ where $r(\cdot)$ denotes the spectral radius of its matrix argument (i.e. compute the eigenvalues and then the spectral radius is the largest number resulting from evaluating the modulus of each of these numbers). If, however, they are of the 
form (3) then asymptotic stability holds if, and only if, all solutions in $z$ of

$$
\operatorname{det}\left(z I_{n}-\sum_{j=0}^{\alpha-1} J_{j} z\left(z I_{m}-D_{0}\right)^{-1} C\left[A+B_{0} X_{j}(z)\right]\right)=0
$$

where

$$
X_{j}(z)=\left[\left(z I_{m}-D_{0}\right)^{-1} C\right]^{j}
$$

have modulus strictly less than unity.

At this stage, we see that the structure of the pass state initial vector sequence has a crucial effect on the asymptotic (and hence stability along the pass) stability property. In particular, suppose that this sequence is modelled as (2) when it should have been modelled as a particular case of (3). Then the example concerned could be treated as being asymptotically stable when in fact it is not and hence any subsequent analysis will be incorrect.

In computational terms, it is easy to check asymptotic stability for the case when (2) is the pass state initial vector sequence. If, however, we must use (3) then (4) is not a suitable starting point and also provides no insight into how (if at all) control action could be employed to guarantee asymptotic stability (and hence meaningful onward analysis). The rest of this section shows how this problem can be overcome using the so-called behavioral approach and, in particular, that specialized for application to discrete linear repetitive processes (Sule and Rogers 2004).

The behavioral approach begins by constructing an equivalent 1D discrete linear systems state-space equivalent model of the repetitive dynamics. This idea has been used in the analysis of $2 \mathrm{D} / \mathrm{nD}$ linear systems (e.g. Aravena et al. 1990) but the finite pass length property of discrete linear repetitive processes releases further structure which can be exploited in analysis.

Introduce the following vectors defined from the vector variables in (1) (where col denotes a column vector with compatible dimension)

$$
\begin{aligned}
Y(k) & =\operatorname{col}\left(y_{k}(0), y_{k}(1) \ldots y_{k}(\alpha-1)\right) \\
U(k) & =\operatorname{col}\left(u_{k}(0), u_{k}(1) \ldots u_{k}(\alpha-1)\right) \\
X(k) & =\operatorname{col}\left(x_{k}(0), x_{k}(1) \ldots x_{k}(\alpha-1)\right)
\end{aligned}
$$

and the vector $W(k)$ as

$$
W(k)=\left[\begin{array}{ll}
Y^{T}(k) & U^{T}(k)
\end{array}\right]^{T}
$$

which in the behavioral approach to analysis we treat as the vector valued manifest variable and $X(k)$ is known as the vector valued latent variable. Then (1) and (3) can be rewritten as (taking $d_{k+1}=0, k \geq 0$ without loss of generality)

$$
\left(\left[\begin{array}{ll}
0 & \hat{B} \\
I & 0
\end{array}\right] \sigma+\left[\begin{array}{cc}
\hat{K} & 0 \\
-\hat{D}_{0} & 0
\end{array}\right]\right) W(k)=\sigma\left[\begin{array}{c}
\hat{Q} \\
\hat{C}
\end{array}\right] X(k)
$$

where $\sigma$ denotes the difference operator $\sigma f(k)=f(k+1)$ on sequences $f(k)$. The matrices in the above equation are given by (where diag denotes a block diagonal matrix of compatible dimensions)

$$
\begin{aligned}
\hat{D_{0}} & =\operatorname{diag}\left\{D_{0}, D_{0}, \ldots D_{0}\right\} \\
\hat{C} & =\operatorname{diag}\{C, C, \ldots C\}
\end{aligned}
$$




$$
\begin{aligned}
& \hat{B}=\left[\begin{array}{ccccc}
0 & & & & \\
B & 0 & & & \\
& B & 0 & & \\
& & \ddots & \ddots & \\
& & & B & 0
\end{array}\right], \hat{Q}=\left[\begin{array}{rrrrr}
I & & & \\
-A & I & & \\
& -A & & \\
& & \ddots & \ddots & \\
& & & -A & I
\end{array}\right] \\
& \hat{K}=\left[\begin{array}{ccccc}
J_{0} & J_{1} & \ldots & \ldots & J_{\alpha-1} \\
B_{0} & & & & \\
& B_{0} & & & \\
& & \ddots & & \vdots \\
& & & B_{0} & 0
\end{array}\right]
\end{aligned}
$$

This completes the so-called latent variable (or hybrid) representation of the dynamics of the discrete linear repetitive processes considered here. In particular, we have a linear time invariant discrete time behavior with a finite number of manifest variables which are the elements in the vector $W(k)$.

In (5) the matrices are defined over the polynomial ring $\mathbb{R}[\sigma]$ and let the collection of all sequences $f(k), k=0, \ldots, f(k) \in \mathbb{R}$ be denoted by $\mathcal{V}$. Then $\mathcal{V}$ is a module over the commutative ring $\mathbb{R}[\sigma]$ under the operation $\sigma f(k)=f(k+1)$. The entries in the solution trajectories of $W(k)$ and $X(k)$ are also such sequences. Hence the behavior of $W$ is also defined as a module over this ring. This allows all the techniques of behavioral theory to be applied since the manifest and latent variables are defined over a finite Cartesian product of the module $\mathcal{V}$.

It is not required to give all elements of the behavioral theory for this case and instead we can move to the following result which gives the kernel representation and is central to the rest of this paper.

Lemma 1 (Sule and Rogers 2004) The behavior of $W(k)$ has kernel representation

$$
\left(\sigma I+\left(Q_{0} \hat{K}-\hat{D}_{0}\right) \quad \sigma Q_{0}\right) W(k)=0
$$

where

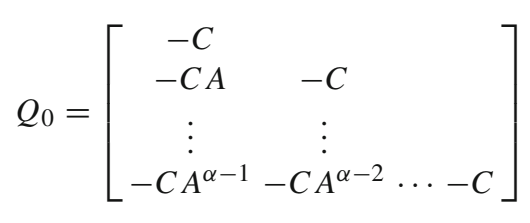

In the behavioral setting, stability (asymptotic stability) of a behavior is characterized by uniform boundedness (asymptotic decay) of all of its trajectories and is hence only applicable to behaviors which are autonomous i.e. those which do not have free (or input) variables since such variables can always be chosen as unbounded. The following result is Theorem 3 in Sule and Rogers (2004).

Theorem 1 A discrete linear repetitive process described by (1) and (3) is asymptotically stable if, and only if,

$$
r\left(\hat{D}_{0}-Q_{0} \hat{K}\right)<1
$$


At this stage, we have reduced the problem of determining the asymptotic stability property of a process described by (1) and (3) to the same task as that for a process with (3) replaced by (2), i.e. computing the eigenvalues of a matrix with constant entries. Next we proceed to show that the existence of a control law to stabilize an asymptotically unstable process described by (1) and (3) is equivalent to the standard discrete linear systems pole placement problem.

\section{Stabilizing control law design}

Rewrite (7) in the form

$$
Y(k+1)=-M_{0} Y(k)-N_{0} U(k)
$$

where $M_{0}=Q_{0} \hat{K}-\hat{D_{0}}, N_{0}=Q_{0} \hat{B}$ and consider the application of the control law

$$
U(k)=K Y(k)
$$

to give

$$
Y(k+1)=-\left(M_{0}+N_{0} K\right) Y(k)
$$

and this controlled process is asymptotically stable if, and only if, $r\left(-M_{0}-N_{0} K\right)<1$. Moreover, a $K$ exists provided the pair $\left\{M_{0}, N_{0}\right\}$ is controllable. This control law is activated by the previous pass profile (recall the definition of the entries in the vector $Y$ ) which is the process output and hence has already been computed. Here we require it to be stored (for one complete pass) and we also assume that it is not corrupted by noise etc.

Controllability of the pair $\left\{M_{0}, N_{0}\right\}$ (which here is also equivalent to reachability) requires that

$$
\operatorname{rank}\left[z I+M_{0} \mid-N_{0}\right]=\alpha m, \quad \forall|z| \in \mathbb{C}
$$

A detailed investigation of controllability for discrete linear repetitive processes with dynamic boundary conditions can be found in Rogers et al. (2002).

The control law design problem has now been reduced to the 1D discrete linear systems pole placement problem. In particular, this problem has a solution if, and only if, there exists a symmetric positive-definite matrix $P$, written $P \succ 0$, such that

$$
\hat{A}^{T} P \hat{A}-P \prec 0
$$

where $\hat{A}=-M_{0}-N_{0} K$. Now apply the well known Schur's complement formula to this last condition and then pre- and post-multiply the result by $\operatorname{diag}\{I, P\}$ to obtain

$$
\left[\begin{array}{cc}
-P & \hat{A}^{T} P \\
P \hat{A} & -P
\end{array}\right] \prec 0
$$

or, equivalently,

$$
\left[\begin{array}{cc}
-P & \left(-M_{0}-N_{0} K\right)^{T} P \\
P\left(-M_{0}-N_{0} K\right) & -P
\end{array}\right] \prec 0
$$

Pre- and post-multiplying this last condition by $\operatorname{diag}\left\{I, P^{-1}\right\}$ now yields

$$
\left[\begin{array}{cc}
-P^{-1} & P^{-1}\left(-M_{0}-K N_{0}\right)^{T} \\
\left(-M_{0}-N_{0} K\right) P^{-1} & -P^{-1}
\end{array}\right] \prec 0
$$


Now let $X=P^{-1}, \hat{Y}=K P^{-1}, R_{0}=-N_{0}$. Then solving the following LMI for the unknowns $X$ and $\hat{Y}$

$$
\left[\begin{array}{cc}
-X & -X M_{0}^{T}+\hat{Y}^{T} R_{0}^{T} \\
-M_{0} X+R_{0} \hat{Y} & -X
\end{array}\right] \prec 0
$$

gives $K=\hat{Y} X^{-1}$ as the stabilizing control law matrix.

As a numerical example to highlight the critical role of the boundary conditions and, in particular, the initial pass state vector sequence consider the case when $\alpha=9$ and

$$
\begin{gathered}
A=\left[\begin{array}{cc}
0.5 & 0 \\
0 & 0.4
\end{array}\right], \quad B=\left[\begin{array}{ccc}
1 & 0 & 1 \\
0 & 0.5 & 0
\end{array}\right], \quad B_{0}=\left[\begin{array}{llll}
1 & 0 & 0 & 0 \\
0 & 1 & 0 & 1
\end{array}\right], \\
C=\left[\begin{array}{ll}
1 & 0 \\
0 & 1 \\
0 & 0 \\
0 & 1
\end{array}\right], \quad D_{0}=\left[\begin{array}{cccc}
0.5 & 0 & 0 & 0 \\
0 & 0.4 & 0 & 0 \\
0.3 & 0 & 0.6 & 0 \\
1 & 0.5 & 1 & 0.2
\end{array}\right] .
\end{gathered}
$$

If the initial pass state vector is described by (2) then it follows immediately that the process is asymptotically stable since $r\left(D_{0}\right)<1$. Suppose now that the initial pass state vector is of the form (3) with

$$
\begin{array}{lll}
J_{0}=\left[\begin{array}{cccc}
0.2 & 0 & 0 & 0 \\
-0.1 & 0 & 0 & 0
\end{array}\right], & J_{1}=\left[\begin{array}{llll}
-0.2 & 0 & 0 & 0 \\
-0.2 & 0 & 0 & 0
\end{array}\right] \\
J_{2}=\left[\begin{array}{llll}
0 & -0.1 & 0 & 0 \\
0 & -0.1 & 0 & 0
\end{array}\right], & J_{3}=\left[\begin{array}{llll}
0 & 0 & 0.1 & 0 \\
0 & 0 & 0.1 & 0
\end{array}\right]
\end{array}
$$

and $J_{j}=0, j=4, \ldots, 8$. Then a routine eigenvalue calculation shows that this process is asymptotically unstable and we now proceed to design a control law to ensure this property. On completion of this we have Figs. 1 and 2 which are the uncontrolled and controlled responses in Channel 3 (the others show the same conclusion and are hence omitted here) respectively and clearly demonstrate that asymptotic stability has been achieved (but further attention may be required in terms of the transient dynamics in the along the pass direction).

\section{Stabilizing boundary conditions}

Consider again a process described by (1) and (2). Then achieving asymptotic stability is only part of the overall task and it may well be that a control law designed for asymptotic stability conflicts with the requirements for stability along the pass and other performance specifications. In fact, the absence of an explicit contribution from the input $u_{k+1}(p)$ in the equation for the pass profile $y_{k+1}(p)$ in (1) makes this situation highly likely (recall again that asymptotic stability is completely determined by the matrix $D_{0}$ ). In such a case, it may be necessary to invoke a multi-loop strategy and in this section we show how to achieve asymptotic stability without using the control input vector $u_{k+}(p)$. The route is to use a pass state initial vector sequence of the form (3) (with $d_{k+1}=0, k \geq 0$ ), i.e. a form of boundary control as in the theory of partial differential equations. 


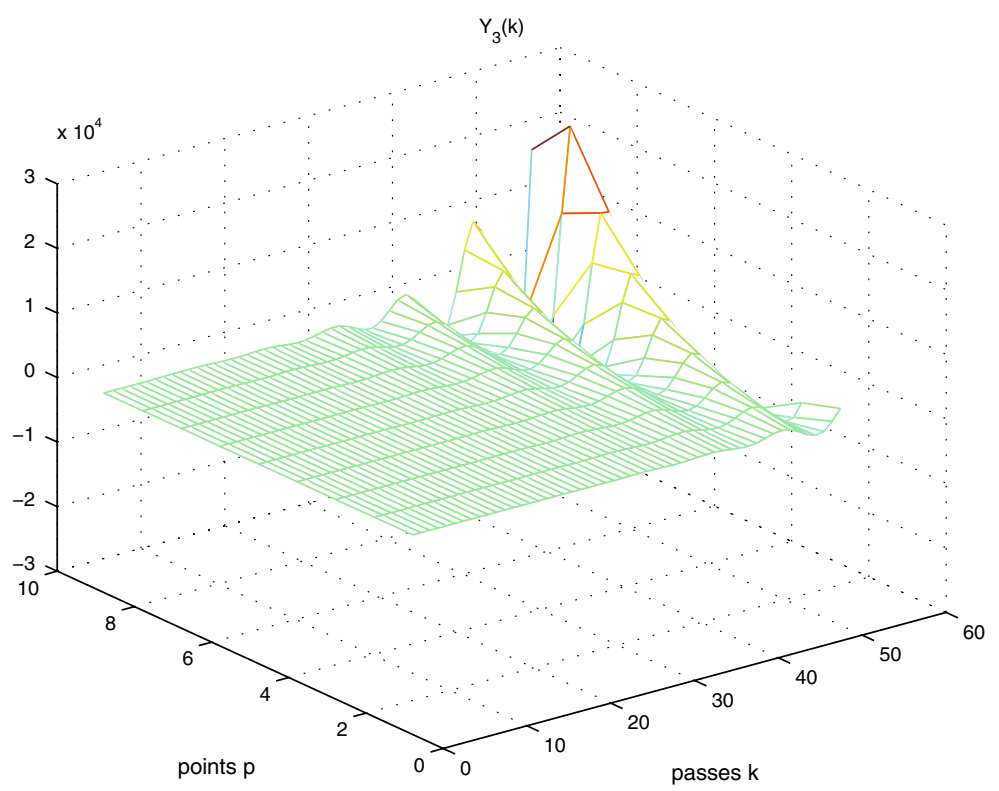

Fig. 1 Uncontrolled response in Channel 3

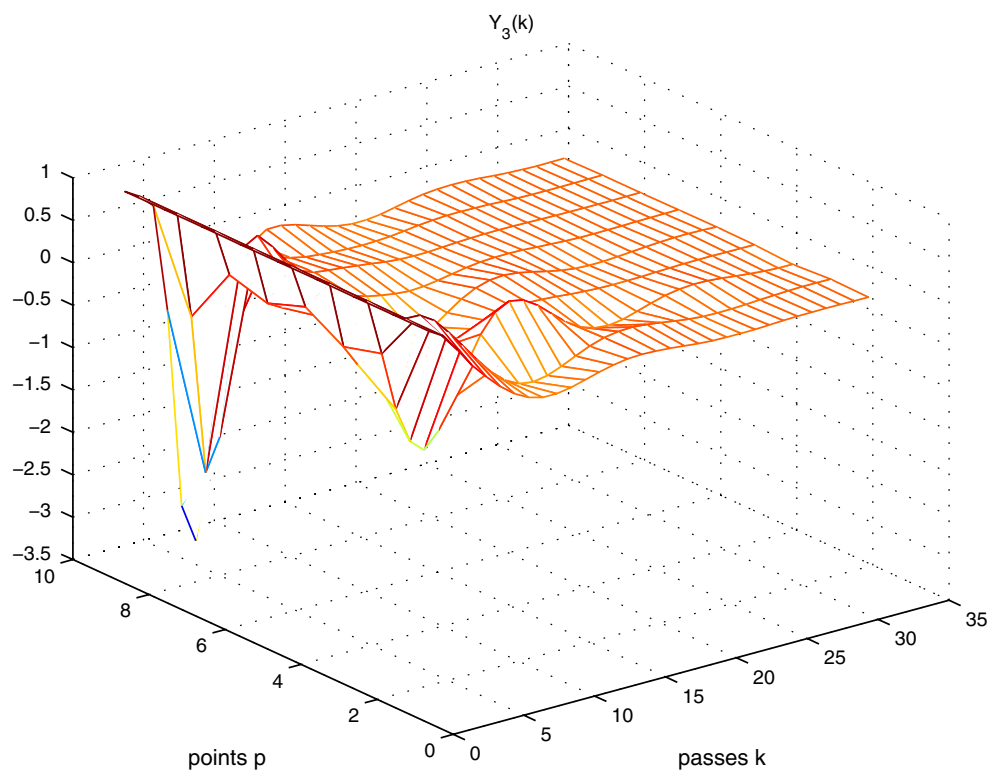

Fig. 2 Controlled response in Channel 3 
Let

$$
\bar{B}_{0}=\left[\begin{array}{ccccc}
0 & 0 & \ldots & \ldots & 0 \\
B_{0} & & & & \\
& B_{0} & & & \\
& & \ddots & & \vdots \\
& & & B_{0} & 0
\end{array}\right] \text { and } \bar{J}=\left[\begin{array}{ccccc}
J_{0} & J_{1} & \ldots & \ldots & J_{(\alpha-1)} \\
0 & & & & \\
& 0 & & & \\
& & \ddots & & \vdots \\
& & & 0 & 0
\end{array}\right]
$$

and hence the matrix $\hat{K}$ of (6) can be written as $\hat{K}=\bar{B}_{0}+\bar{J}$. Consequently we can write

$$
Y(k+1)=(\bar{A}+\bar{B} \bar{J}) Y(k)-N_{0} U(k)
$$

where $\bar{A}=-Q_{0} \bar{B}_{0}+\hat{D}_{0}$ and $\bar{B}=-Q_{0}$.

The control law design problem is now to chose $\bar{J}$ such that

$$
r(\bar{A}+\bar{B} \bar{J})<1
$$

Hence, with the assumption that the pair $\{\bar{A}, \bar{B}\}$ is controllable, and routine modifications to the LMI analysis of the previous section which are omitted here, we have the requirement that

$$
\left[\begin{array}{cc}
-X & X \bar{A}^{T}+\bar{Y}^{T} \bar{B}^{T} \\
\bar{A} X+\bar{B} \hat{Y} & -X
\end{array}\right] \prec 0
$$

where $\hat{X}=P^{-1}$ and $\hat{Y}=\bar{J} X$. Also if this LMI is satisfied the control law matrix is given by $\bar{J}=\hat{Y} X^{-1}$. Note here that the current pass input vector (in the form of $U(k)$ in this representation) plays no role in the stabilization problem.

As a numerical example, consider the case when

$$
\begin{array}{r}
A=\left[\begin{array}{cc}
0.9 & 0 \\
0 & 0.9
\end{array}\right], \quad B=\left[\begin{array}{ccc}
1 & 0 & 1 \\
0 & 0.5 & 0
\end{array}\right], \quad B_{0}=\left[\begin{array}{llll}
1 & 0 & 0 & 0 \\
0 & 1 & 0 & 1
\end{array}\right] \\
C=\left[\begin{array}{ll}
1 & 0 \\
0 & 1 \\
0 & 0 \\
0 & 1
\end{array}\right], \quad D_{0}=\left[\begin{array}{cccc}
1.5 & 0 & 0 & 0 \\
0 & 0.4 & 0 & 0 \\
0.3 & 0 & 0.6 & 0 \\
1 & 0.5 & 1 & 0.2
\end{array}\right]
\end{array}
$$

with $\alpha=9$. This example is asymptotically unstable under the boundary conditions $d_{k+1}=0$, $y_{0}(p)=1,0 \leq p \leq 8, k \geq 0$ since $r\left(D_{0}\right)=1.5$. Completing the above design shows that (13) holds in the case when

$$
\begin{aligned}
& J_{0}=\left[\begin{array}{cccc}
-1.12 & 0.00906 & -0.00612 & 0.0138 \\
-0.0146 & -1.09 & -0.0153 & -1.08
\end{array}\right], \quad J_{1}=\left[\begin{array}{cccc}
-1.25 & 0.0363 & -0.0138 & 0.0464 \\
-0.0731 & -1.1 & -0.0662 & -1.05
\end{array}\right] \\
& J_{2}=\left[\begin{array}{cccc}
-1.39 & 0.0676 & -0.0102 & 0.0744 \\
-0.179 & -0.954 & -0.138 & -0.853
\end{array}\right], \quad J_{3}=\left[\begin{array}{cccc}
-1.53 & 0.0773 & 0.00373 & 0.0737 \\
-0.282 & -0.674 & -0.179 & -0.547
\end{array}\right] \\
& J_{4}=\left[\begin{array}{cccc}
-1.68 & 0.0594 & 0.0147 & 0.0485 \\
-0.317 & -0.368 & -0.156 & -0.262
\end{array}\right], \quad J_{5}=\left[\begin{array}{cccc}
-1.79 & 0.0311 & 0.0147 & 0.0209 \\
-0.27 & -0.147 & -0.0932 & -0.0873
\end{array}\right] \\
& J_{6}=\left[\begin{array}{cccc}
-1.7 & 0.0106 & 0.00812 & 0.00541 \\
-0.18 & -0.0403 & -0.0374 & -0.0182
\end{array}\right] \\
& J_{7}=\left[\begin{array}{cccc}
-1.22 & 0.00206 & 0.00242 & 0.000624 \\
-0.0926 & -0.00661 & -0.0095 & -0.00185
\end{array}\right]
\end{aligned}
$$




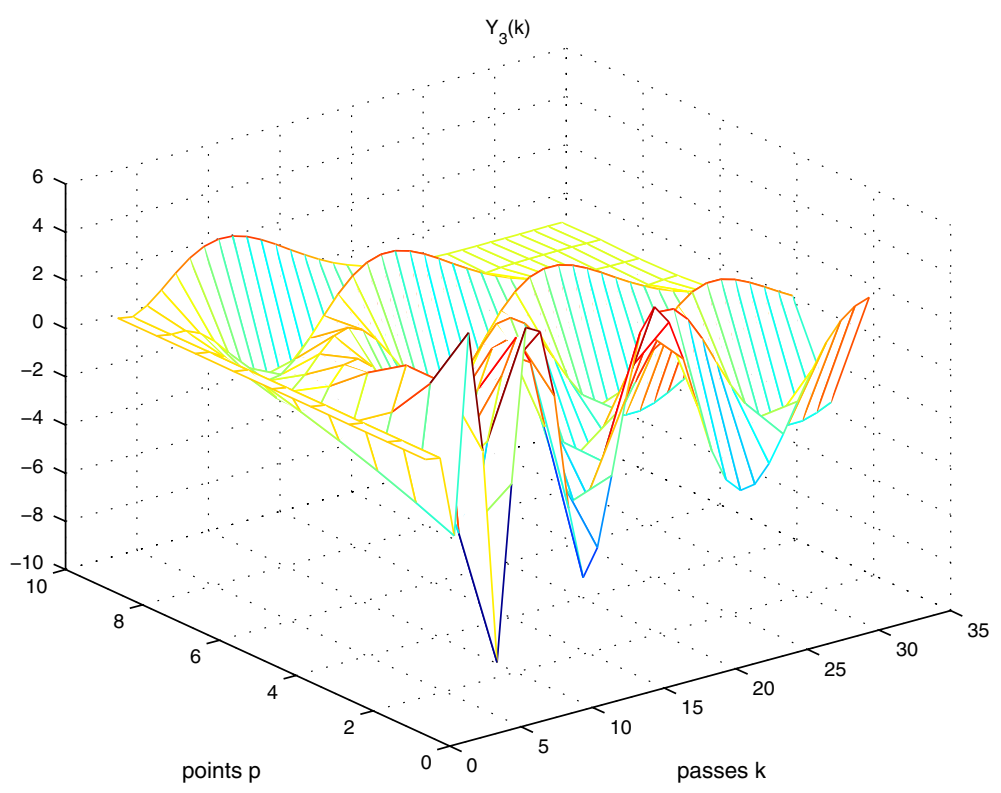

Fig. 3 Controlled response in Channel 3

$$
J_{8}=\left[\begin{array}{cccc}
-0.481 & 0.00015 & -0.000312 & -8.23 \cdot 10^{-6} \\
-0.0303 & -0.000473 & -0.00146 & -2.69 \cdot 10^{-5}
\end{array}\right]
$$

On completion of this we have Fig. 3 which shows the controlled response in Channel 3 (the others show the same conclusion and are hence omitted here) and clearly asymptotic stability has been achieved.

\section{Conclusions}

This paper has considered the influence of the boundary conditions on the stability and control properties of discrete linear repetitive processes. In particular, the case when the pass state initial vector sequence is an explicit function of the previous pass profile has been studied. The presence of such boundary conditions can cause the process to be asymptotically unstable (the weakest form of stability property) and the major new results in this paper show how to design a control law to ensure this property. This control law is feed forward in the sense that it is fully activated by the previous pass profile.

A second feature examined here relates to the fact that control law design for asymptotic stability may conflict with other essential requirements, e.g. stability along the pass and tracking performance. In such cases it may be necessary to consider a multi-loop control strategy. Here we have developed a solution where control is introduced through the pass state initial vector sequence, i.e. a form of boundary control as in partial differential equation (or distributed parameter) control. One area to be investigated in further work is that of minimizing the number previous pass points required for this task, i.e. can we use a control law matrix which has some (but not all) zero sub-blocks and hence a more efficient implementation? 
A natural question to ask is if it is possible to undertake a robustness analysis? The answer with the approach used here is negative due to the fact the block matrices $M_{0}$ and $N_{0}$ contain different products of matrices which prevent effective progress. Hence the development of alternative settings for a robustness analysis of the processes considered here is an obvious subject for further research. This again highlights the critical role of the boundary conditions and, in particular, the pass state initial vector sequence in the analysis and control of discrete linear repetitive processes since if they are of the form (2) a well developed robust control theory is available-see Rogers et al. (2007) for the details. One possible approach here would be to try to exploit the structure of so-called wave repetitive processes (see Rogers et al. (2007) for the definition and currently available results) as a setting for analysis.

\section{References}

Aravena, J. L., Shafiee, M., \& Porter, W. A. (1990). State models and stability for 2-d filters. IEEE Transactions on Circuits and Systems, 37(12), 1509-1519.

Edwards, J. B. (1974). Stability problems in the control of multipass processes. Proceedings of the Institution of Electrical Engineers, 121(11), 1425-1431.

Moore, K. L., Chen, Y., \& Bahl, V. (2005). Monotonically convergent iterative learning control for linear discrete-time systems. Automatica, 41(9), 1529-1537.

Owens, D. H., \& Rogers, E. (1999). Stability analysis for a class of 2D continuous-discrete linear systems with dynamic boundary conditions. Systems and Control Letters, 37, 55-60.

Roberts, P. D. (2000). Numerical investigations of a stability theorem arising from 2-dimensional analysis of an iterative optimal control algorithm. Multidimensional Systems and Signal Processing, 11(1/2), 109-124.

Rogers, E., Galkowski, K., Gramacki, A., Gramacki, J., \& Owens, D. H. (2002). Stability and controllability of a class of 2D systems with dynamic boundary conditions. IEEE Transactions on Circuits and Systems, Part I: Fundamental Theory and Applications, 49(2), 181-195.

Rogers, E., Galkowski, K., \& Owens, D. H. (2007). Control systems theory and applications for linear repetitive processes, volume 349 of Lecture Notes in Control and Information Sciences. Springer-Verlag.

Rogers, E., \& Owens D. H. (1992). Stability analysis for linear repetitive processes, volume 175 of Lecture Notes in Control and Information Sciences. Springer-Verlag.

Sule, V. R., \& Rogers E. (2004). A behavioral approach to the control of discrete linear repetitive processes. Systems and Control Letters, 53, 79-88.

\section{Author Biographies}

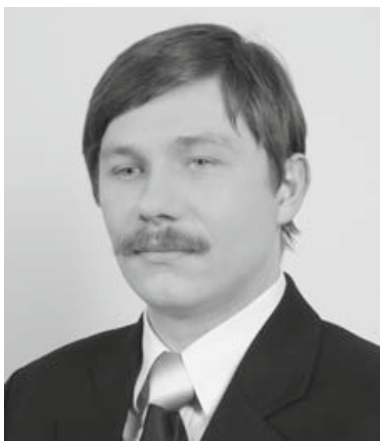

Lukasz Hladowski received the M.Sc degree from the University of Zielona Gora, Poland in 2002. Currently he is with the Institute of Control and Computation Engineering in the University of Zielona Gora. His research interests include the control theory and multidimensional systems, especially the Linear Repetitive Processes. 


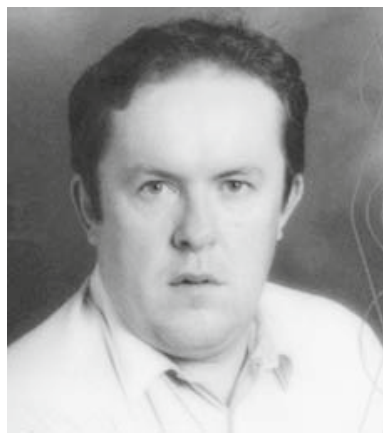

Eric Rogers was born in Northern Ireland and studied Mechanical Engineering as an undergraduate at The Queen's University Belfast and then did masters and $\mathrm{PhD}$ research in Control Systems at The University of Sheffield, UK. In 2004, he was awarded the DSc degree by The Queen's University of Belfast in 2004 for research in Control Systems. He has been a member of the academic staff of The University of Southampton, UK since 1990 and is currently Professor of Control Systems Theory and Design in the School of Electronics and Computer Science. His research interests are in iterative learning and repetitive control and applications, multidimensional systems, processes with repetitive dynamics, and flow control. Recently the iterative learning control research has expanded to include its use in stroke rehabilitation. He is the editor-in-chief of The International Journal of Control.

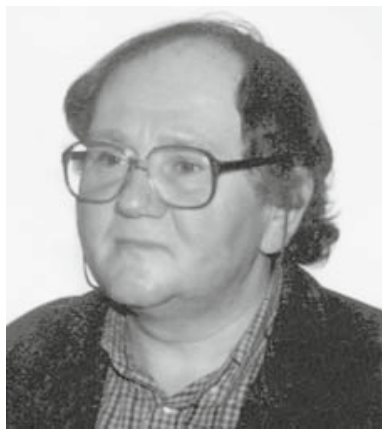

Krzysztof Galkowski received the M.S., Ph.D. and Habilitation (D.Sc.) degrees in electronics/automatic control from Technical University of Wroclaw, Poland in 1972, 1977 and 1994, respectively. In October 1996 he joined the Technical University of Zielona Góra (now the University of Zielona Góra), Poland where he holds the professor position, and he is a visiting professor in the School of Electronics and Computer Science, University of Southampton, UK. In 2002, he was awarded the degree "Professor of Technical Science" the highest scientific degree in Poland. He is an associate editor of International Journal of Multidimensional Systems and Signal Processing, International Journal of Control and International Journal of Applied Mathematics and Computer Science. He has co-organised 4 International Workshops NDS 1998-Lagov, Poland, NDS 2000_Czocha Castle-Poland, the third as a Symposium within MTNS 2002 in Notre Dame IN, US, and the last one NDS 2005 in Wuppertal, Germany. In 2007 NDS 2007 is organized in Aveiro, Portugal. His research interests include multidimensional (nD) systems and repetitive processestheory and applications, control and related numerical and symbolic algebra methods. He is an author/editor of four monographs/books and about 60 papers in peer reviewed journals and over 100 in the proceedings of international conferences. He leads an active group of young researchers in the $\mathrm{nD}$ systems area with strong international co-operation with the Universities of Southampton, for which he is awarded the official position of Visiting Professor at The School of Electronics and Computer Science, and Sheffield UK; the universities of Wuppertal, Erlangen-Nurnberg, Magdeburg, Germany; University of Hong Kong, University of Poitiers, IRIA, Sophia Antipolis, France. He is spending (spent) the academic year 2006-07 (2004/2005) as a Gerhard Mercator guest professor, funded by DFG, in The University of Wuppertal, Germany. In 2004, he obtained a Siemens Award for his research contributions.

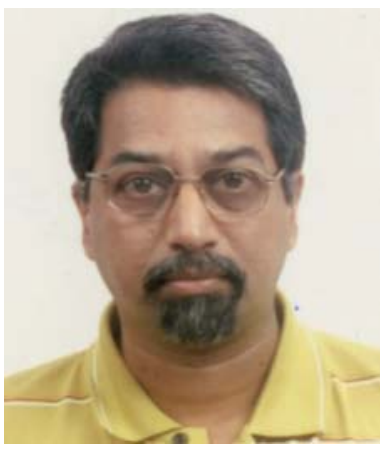

Virendra R. Sule earned the Ph.D. degree at the Indian Institute of Technology Bombay India in 1990. He subsequently was in the faculty of Electrical Engineering at IIT Kanpur and Bombay India until May 2007. His research work has been primarily in Mathematical Systems Theory and has published several papers in internationally refereed journals. Since 2003 he has been working in the field of cryptology. He currently works with Computational Research Laboratories Ltd., Pune, India and is working on cluster computing of computational problems of Number Theory and Algebra. 